\title{
Does PET/CT give incremental staging information in cancer oesophagus compared to CECT?
}

\author{
Gihan Hassan Gamal
}

\begin{abstract}
Background: The purposes of this study were to evaluate the efficiency of (18F-FDG) positron emission tomography/computed tomography (PET/CT) in diagnosing and staging of esophageal cancer and to compare the results with that of contrast-enhanced computed tomography (CECT) using the histopathological diagnosis as a gold standard.
\end{abstract}

Results: This is a prospective study of 19 patients from June 2017 to June 2019 (17 male, 2 female) with mean age of 68 years with newly clinical, and pathologically proven esophageal carcinoma were included in this study, all patients underwent CECT followed by PET/CT imaging as an attempt of proper staging of carcinoma. It was found that PET/CT has changed the stage group of 8 patients out of 19. Six of 8 patients were upstaged on the basis of PET/CT findings while 2 of 8 were downstaged.

The statistical results of CECT in regional lymph nodes sensitivity, specificity, PPV, NPV, and accuracy were 53\%, 95\%, $82 \%, 80 \%$, and $82 \%$ while those of PET/CT were $68 \%, 82 \%, 68 \%, 82 \%$, and $79 \%$, respectively. As regards the distant metastasis, the sensitivity, specificity, PPV, NPV, and accuracy of PET/CT were $100 \%, 83 \%, 96 \%, 100 \%$, and $96 \%$ versus $73 \%, 100 \%, 100 \%, 50 \%$, and $79 \%$ for CECT.

Conclusion: As an important diagnostic method, FGD-PET/CT showed a great impact on initial tumor staging of the patient with esophageal cancer and on the ability to detect distant metastases missed by CECT. Thus, leading to a change in clinical management of a significant number of patients.

Keywords: Esophageal cancer, 18F-fluorodeoxyglucose positron emission tomography/computed tomography (18F-FDG PET/CT), Contrast-enhanced computed tomography (CECT), Standard uptake value (SUV)

\section{Background}

Esophageal carcinoma is one of the leading causes of mortality. It is the sixth leading cause worldwide, principally because of its early dissemination [1].

Imaging examination is an important method in diagnosing and staging esophageal cancer. Conventional staging investigation (CSI) includes barium swallow and endoscopic ultrasound (EUS) which were also used to define the location and the extent of primary tumor. EUS plays a role to assess peri-esophageal and locoregional lymph nodes involvement and extension of the lesion through the esophageal wall, but it has its limitations, as esophageal obstruction by the tumor makes passage of the endoscope beyond the tumor nearly

Correspondence: dr.gh_006@yahoo.com

Associate Professor of Radiodiagnosis, October 6 University, Giza, Egypt impossible and additionally, it is not useful in detecting metastatic diseases [2].

The imaging study by CECT is being used to demonstrate the morphological characters of the lesions. It allows for the assessment of local tumor invasion and providing information regarding distal metastatic disease.

Fluorodexoyglucose (FDG) and positron emission tomography (PET/CT) can improve the delineation of primary tumor volume and involved regional lymph nodes as well as distant metastasis in patients with esophageal cancer as it detects early changes in tumor cell proliferation that precedes morphological changes in the tumor; thus, PET/CT can provide great impact on the pre-treatment evaluation, diagnosis, and staging of patient with esophageal cancer [3]. 
The esophageal cancer staging is defined by the American Joint committee on cancer (AJCC) staging system [4] as the earliest stage esophageal cancer is called stage 0 (high-grade dysplasia). It then ranges from stage I through stage IV. For those with early stage, curative treatment options include surgery or definitive chemoradiation. However, in locally advanced disease, neoadjuvant chemotherapy or chemoradiation is often used in combination with surgery in an attempt to augment the poor survival rate of the disease. Patients with metastatic disease require strategies that provide the best chance for maintaining quality of life [5].

Patients with stage III have cancer that invades through the wall of the esophagus and has spread to the adjacent lymph nodes and or invaded adjacent structures. This is a very common stage for the presentation of esophageal cancer. Stage III cancer may also be referred to as a locally advanced disease. Stage IV means that the cancer has metastasized or has spread to other parts of the body [6].

The aim of this study was to evaluate the utility of FDG-PET/CT in staging esophageal cancer and comparing the results to CECT using histologic findings as a gold standard in an attempt to determine whether PET/ CT adds additional information over CECT in staging patients with esophageal cancer.

\section{Methods}

\section{Patients selection and preparation}

This prospective study was conducted on 19 patients over a period starting from June 2017 to June 2019. Seventeen males and 2 females with a mean age of 68 years pathologically proved to have esophageal cancer. All patients underwent a routine evaluation which includes history, physical examination, blood sugar level, and barium swallow. EUS was done for some patients in special cases when it was needed.

Patients with a fasting glucose level of $200 \mathrm{mg} / \mathrm{dL}$ were excluded (exclusion criteria) from the study.

\section{The CECT technique}

All patients underwent CECT with Somatom Plus 4 spiral CT scanner of Siemens Medical system. A 300$400 \mathrm{ml}$ of oral contrast and iodinated IV contrast medium were first given to the patient, and the following parameters used were $80-100 \mathrm{~mA}, 140 \mathrm{kV}, 5-\mathrm{mm}$ collimation, $0.5 \mathrm{~s}$ rotation time; and pitch $=0.984$. Patients were in a supine position with both arms up and normal respiration was maintained during the scanning. Reconstruction with a section thickness of $1.2 \mathrm{~mm}$ was done.

The patients were required to lie still on the table throughout the rapid injection of the iodinated contrast media, $50 \mathrm{ml}$ of iopromide. An automatic injector was used over $12.5 \mathrm{~s}$ through an 18-gauge intravenous line placed unto the right antecubital vein at a rate of $4 \mathrm{ml} / \mathrm{s}$. Immediately following the injection of the iodinated contrast, $50 \mathrm{ml}$ saline was fused by the same injector and via the same route. Thirty seconds after the start of the injection, scans were performed starting from the base of the skull down to the mid-thigh. Through the chest in an arterial vascular phase, upper abdomen in the portal phase and the stomach should be distended by water as a contrast agent to determine the extent of the gastric involvement.

The interpretation of images concentrated on the following criteria: the thickness of the esophageal wall, site and extent of the primary tumor, regional lymph node, invasion of adjacent structures, and distant nodal and organ metastases.

CECT images were interpreted by an experienced radiology consultant and reports were compared to those of $\mathrm{PET} / \mathrm{CT}$. The reader was not blinded to other modality results.

\section{The PET/CT technique}

18F-FDG PET/CT scans were obtained using PET/CT scanner (Discovery STE; GE Healthcare, Boston, USA).

All patients underwent this technique after at least 2 weeks from completion CECT. CT component was done with oral and without IV contrast, with a voltage peak of $140 \mathrm{kV}, 80 \mathrm{MAs}$, slice thickness of $5 \mathrm{~mm}$ and rotation speed of $0.8 \mathrm{~s} /$ rotation. Our imaging protocol required patients to fast $6 \mathrm{~h}$ before injection of 18F-FDG (5 MBq/ $\mathrm{kg}$ ) up to a maximum of $400 \mathrm{MBq}$, and data acquisition started 45-60 min after injection of 18F-FDG for the whole-body scan from the base of the skull down to the mid-thigh at 2 min per bed position. Both CT and PET scans were obtained during normal tidal breathing.

The standard uptake value (SUV) was defined as the tissue concentration $(\mathrm{MBq} / \mathrm{mL})$ of the tracer divided by the activity injected per body weight $(\mathrm{MBq} / \mathrm{g})$. The maximum SUV in the volume of interest was considered as the SUVmax for the purpose of analysis. PET images were reconstructed with $\mathrm{CT}$ images.

CT images and fused PET/CT images were reviewed in axial, coronal, and sagittal planes.

The PET/CT images were interpreted by an experienced radiology consultant and reports were compared to those of CECT. The reader was not blinded to other modality results.

A comparison between CECT and PET/CT findings was validated by pathological analysis of resection specimen.

\section{Image interpretation}

For interpretation of PET-CT scans, we used visual inspection (identified as 18F-FDG-avid foci in tumor or lymph nodes typically greater than the adjacent normal 

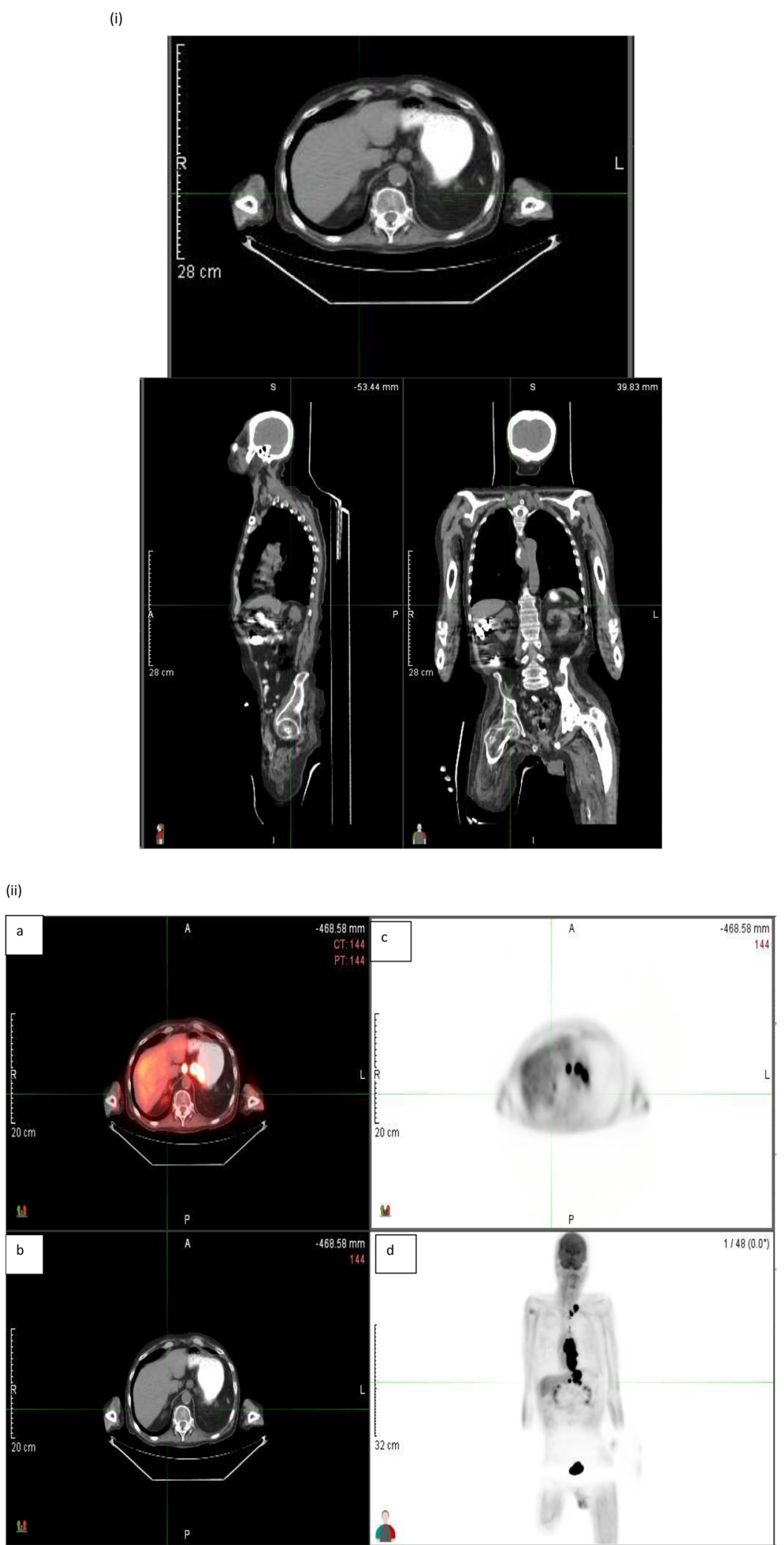

Fig. 1 A 78-year-old male. (i) CT. (ii) CT/PT fusion. FDG avid $14.5 \mathrm{~cm}$ long esophageal neoplasm (SUVmax37) with extension to the esophagogastric junction and adjacent gastric cardia. Metastatic periosophageal lymphadenopathy. Small right adrenal metastasis .Paget's disease involving the left hemipelvis and proximal left femur. a Axial PET/CT. b Axial CT. c and d PET 


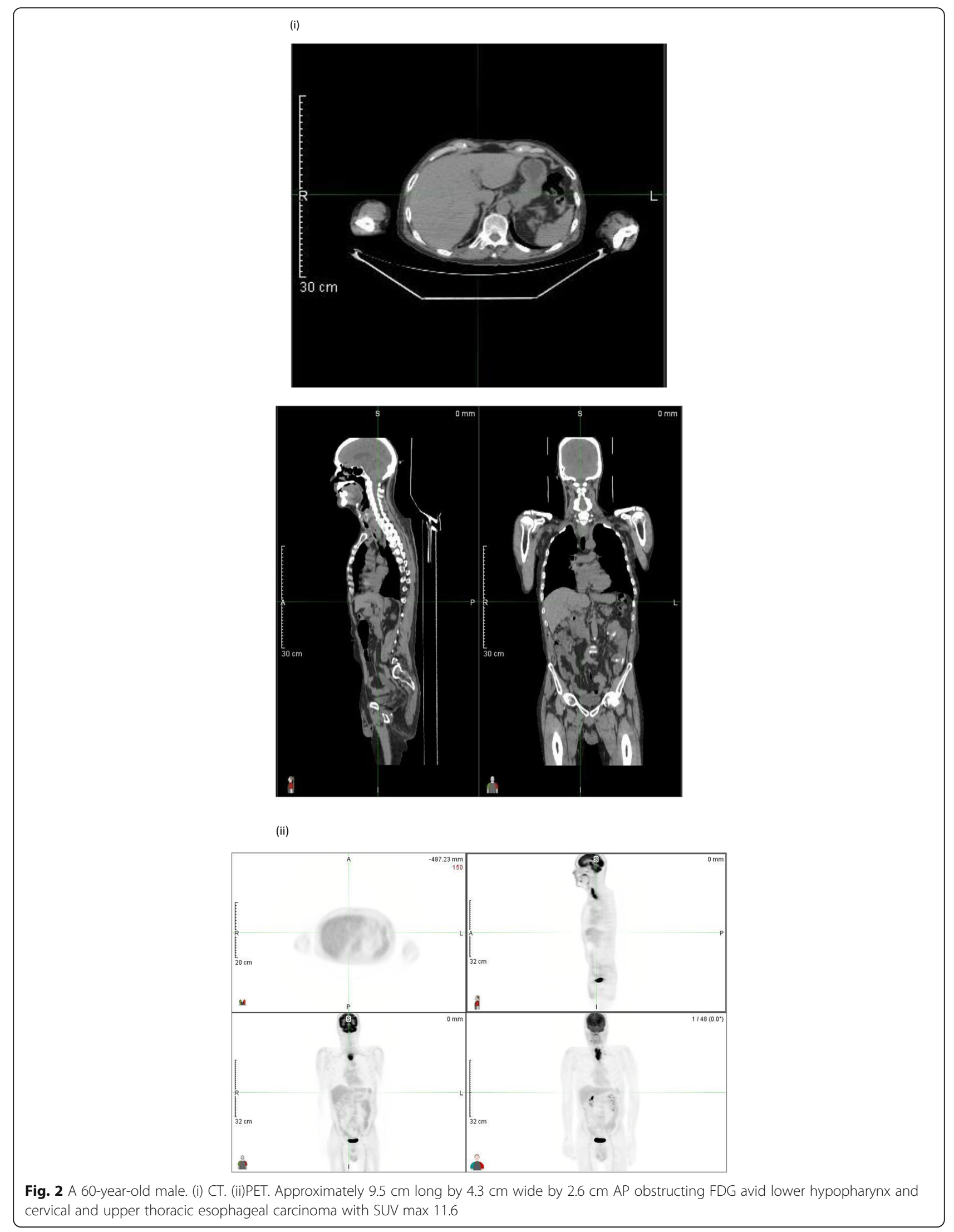


tissue) and standardized uptake value(SUV max) method (Fig. 1).

The interpretation of CECT scans for the tumor was by estimation of esophageal wall thickness (more than 5 $\mathrm{mm}$ ) and for lymph node when the short-axis diameter was greater than $10 \mathrm{~mm}$.

\section{Statistical analysis}

Sensitivity, specificity, PPV, NPV and accuracy of CECT and PET/CT for detection of the primary tumor, locoregional lymph nodes involvement and distant metastases diseases were calculated and compared using McNemar $T$ test.

Statistical significance was set at $p<0.05$.

The standard maximum uptake value (SUVmax) of primary tumor, locoregional lymph nodes, and distant metastases was quantitative and qualitative evaluated. Data were coded and entered using the statistical package for social sciences SPSS version 22 .

\section{Results}

This prospective study was performed on 19 patients who underwent CECT with histopathologically confirmed esophageal cancer, for pre-operative staging using 18F-FDG PET/CT scanning. Eight patients had adenocarcinoma and 11 patients with squamous cell carcinoma. Two of 19 cases had mid-esophagus involvement and 11 cases had lower-esophagus and 6 gastroesophageal junction involvement. All patients tolerated CECT and PET/CT examinations with no problems or complications. No history of undergone any therapy at the time of the study. The calculated SUVmax of the primary tumors identified by both CECT and PET/CT were ranged from 11.6 to 37.0 (Fig. 2).

Patient and tumor characteristics were showed in Table 1.

The PET/CT was found to have changed the stage group of 8 patients out of 19 (40\%). Six of 8 patients were upstaged on the basis of PET/CT findings while 2 of 8 were downstaged.

The comparison between the CECT and PET/CT stage groups was shown in Table 2.

Eleven patients were unchanged concerning their stage group. Of these 8 patients, PET/CT detects additional regional lymph node and distant nodal metastasis in 6 patients. The remaining 2 patients, PET/CT detects additional distant systemic metastasis at the liver and lung. $\mathrm{PET} / \mathrm{CT}$ correctly excluded distant nodal and systemic metastasis in 3 patients.

Primary tumor was identified by CECT, in 13/19 patients with stage group had T3 tumor and the remaining 6 patients had T4 tumor. While results of PET/CT proved that 12/19 patients had T3 and 7 patients had T4.
Table 1 Patient and tumor characteristics $(n=19)$

\begin{tabular}{llll}
\hline Sex & Male & 17 & $89.5 \%$ \\
& Female & 2 & $10.5 \%$ \\
Histology & Adenocarcinoma & 8 & $42.1 \%$ \\
& Squamous cell carcinoma & 11 & $57.9 \%$ \\
Site of 1ry lesion & Mid-esophagus & 2 & $10.5 \%$ \\
& Lower-esophagus & 11 & $57.9 \%$ \\
& G/E junction & 6 & $31.6 \%$
\end{tabular}

Patients with stage T3 means that cancer invaded through the whole wall of the esophagus and had spread to the adjacent lymph nodes. This was the commonest stage for the presentation of esophageal cancer in this study. It is also referred to as a locally advanced tumor and was considered as resectable tumor, and those with stage T4 where that cancer had spread to other parts of the body and considered as an unrespectable tumor (Fig. 3).

The calculated SUVmax of the primary tumors ranged from 11.6 to 37.0.

As regards regional lymph node involvement, CECT identified $4 / 19$ patients, only 3 were confirmed by pathology and one was false negative. PET/CT detected lymph node involvement in 8/19 patients. Six were proved by pathology and 2 proved to be false positive. The overall sensitivity, specificity, PPV, NPV, and accuracy for the detection of regional lymph nodes were calculated.

The statistical results of CECT in regional lymph nodes, sensitivity, specificity, PPV, NPV, accuracy were $53 \%, 95 \%, 82 \%, 80 \%$, and $82 \%$ while those of PET/CT were $68 \%, 82 \%, 68 \%, 82 \%$, and $79 \%$, respectively. The calculated SUVmax ranged from 4.9 to 6.8 .

As regards distant metastasis, the sites of distal metastases in our study were in the lung, liver, bone, and distant lymph nodes.

CECT identified 11/19 patients with distal metastasis while FDG-PET/CT identified metastasis in 17/19 patients. So, 6 patients were upstaged on the basis of PET/ CT findings, 2 patients with focal hepatic high-uptake lesions and 4 patients with distant LN metastases which were missed by CECT.

Table 2 Comparison of CECT and PET/CT tumor stage group

\begin{tabular}{lll}
\hline Patient no. & CECT staging & PET/CT staging \\
\hline 1. & III & IV \\
2. & III & IV \\
3. & III & IV \\
4. & III & IV \\
5. & III & IV \\
6. & III & IV \\
7. & IV & II \\
8. & IV & II \\
\hline
\end{tabular}




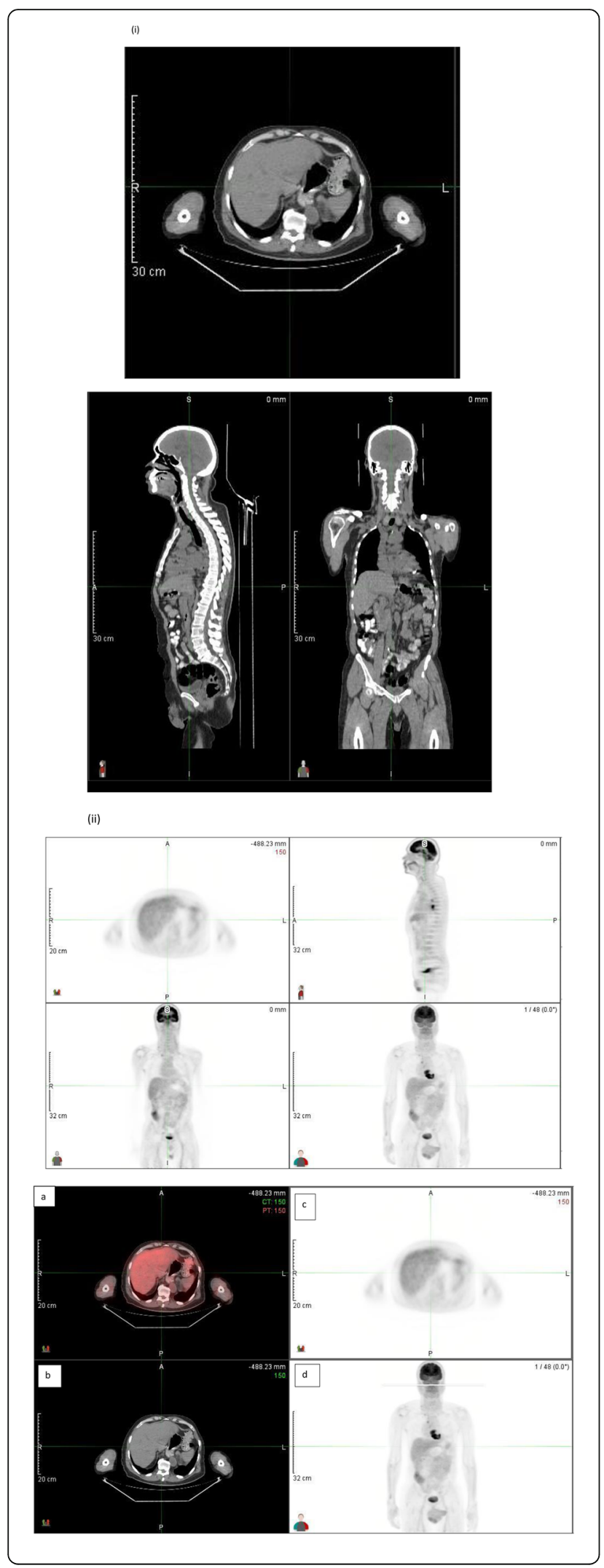

Fig. 3 A 63-year-old male. (i) CT. (ii) PET. (iii) CT/PT fusion. Approximately $5.5 \times 5.0 \times 3.5 \mathrm{~cm}$ distal esophageal/esophagogastric neoplasm with SUV max 12.4. Hyperplastic peri-esophageal and right retrocrural nodes up to $1 \mathrm{~cm}$ are not discretely FDG avid but likely tumor-bearing. FDG avid subcarinal, right retrocaval pre-tracheal, high right paratracheal, and right superior mediastinal nodes up to 2 $\mathrm{cm}$ with SUV max 3.5. No extrathoracic spread of neoplasm. a Axial PET/CT. b Axial CT. c and d PET

Sensitivity, specificity, PPV, NPV, and accuracy of $\mathrm{PET} / \mathrm{CT}$ versus CECT in distant metastatic diseases were calculated for CECT were $73 \%, 100 \%, 100 \%, 50 \%$, and $79 \%$ while those of for PET/CT were $100 \%, 83 \%$, $96 \%, 100 \%$, and $96 \%$, respectively. The calculated SUVmax ranged from 4.8 to 7.0 .

\section{Discussion}

By the advantage of PET/CT to demonstrate the biological function of the tumor before anatomical changes took place, a PET/CT scan enables physicians to more accurately diagnose and stage patients with esophageal cancer thus changing in clinical management of a significant number of patients avoiding unnecessary surgery [7].

In the current study, PET/CT had changed the stage group of 8 patients out of 19 (40\%). Six of 8 patients were upstaged while 2 of 8 were downstaged; this was achieved by its greatest ability to detect distant metastases in 6 patients with esophageal cancer missed by CECT. This results led to the upstaging of these patients from stage III to stage IV.

As a general rule, all stage $0, \mathrm{I}$, and II are considered resectable. Most stage III cancer, which was the commonest stage for presentation of esophageal cancer in this study, are potentially resectable, even when they have spread to the nearby lymph nodes as long as cancer has not grown into important adjacent structures such as the lung, trachea, heart, aorta and spine. Cancer that has spread into these nearby important structures or that has spread to the distant lymph nodes is considered unresectable, so treatment other than surgery is usually the best option [8].

The aforementioned results are in accordance with those previously concluded that the ability of PET/CT to identify otherwise occult metastases has led to alter staging in up to $30 \%$ of esophageal cancer cases $[9,10]$.

The statistical parameters of the current study displayed sensitivity, specificity, PPV, NPV, and accuracy of PET/CT were $100 \%, 83 \%, 96 \%, 100 \%$, and $96 \%$, while those of CECT were $73 \%, 100 \%, 100 \%, 50 \%$, and $79 \%$, respectively in detecting distant metastatic diseases.

As regards the regional lymph node, no significant statistical differences could be detected in the current study between both modalities. This may be due to the small study sample. 
The current study showed sensitivity, specificity, PPV, NPV, and accuracy for PET/CT were $68 \%, 82 \%, 68 \%$, $82 \%$, and $79 \%$ while those of CECT were $53 \%, 95 \%, 82 \%$, $80 \%$, and $82 \%$, respectively.

These do not correspond with the previous studies done by $[11,12]$ as the authors concluded that PET/CT has similar specificity but significantly greater sensitively and accuracy than CECT for detection of LN metastases in staging esophageal cancer.

PET/CT sensitivity, specificity, and accuracy for metastatic LN detection were $52 \%, 94 \%$, and $84 \%$, respectively comparing to $15 \%, 97 \%$, and $77 \%$ of CECT, respectively.

\section{Limitations of the study}

1. This study has its limitations as the staging of esophageal cancer was adapted only on patients with stage T3 which was the commonest stage group of presentation of esophageal cancer in this study.

2. Small sample size which limits its statistical power.

3. Due to limited spatial resolution, PET/CT could result in false negative of regional lymph nodes which are in direct vicinity of the tumor, as the avid uptake by the tumor may obscure the peritumoral node. However, the utility of PET/CT over CECT in regional LN detection needs further studies to improve its diagnostic accuracy.

4. High cost of PET/CT scan.

In addition to PET/CT scans, MRI with the functional feature of diffusion-weighted imaging (DWI) is another advancing imaging technology, which has current and future potential to overcome the limitations of conventional staging methods in patients with esophageal carcinoma [13].

MRI possessed the advantage including multiparameter imaging, lack of ionizing radiation, safer contrast agent, and the ability to determine the functionality of tumors with DWI. The technique is applied in wholebody MRI (WBMRI) DWI. Compared with PET/CT, WBMRI has similar accuracy in detecting the primary tumor, nodal deposits, and metastatic disease. In a study done by [14], the PET-CT and WBMRI detected the primary tumor in 46 of 49 (94\%) and 48 of 49 (98\%) patients, respectively. The sensitivity, specificity, positive predictive value (PPV), negative predictive value (NPV), and accuracy of nodal metastasis detection in patients undergoing surgery $(n=18)$ were $27 \%, 100 \%, 100 \%$, $47 \%$, and $56 \%$ for PET-CT compared with $30 \%, 100 \%$, $100 \%, 53 \%$, and $61 \%$ for WBMRI.

The investigation of WBMRI in esophageal carcinoma is still limited and it is still early to derive a conclusion.
However, the use of WBMRI in systemic evaluation of esophageal cancer has gradually increased [15].

\section{Conclusion}

In conclusion, PET/CT can aid in the management of esophageal cancer patients, providing anatomic and metabolic information thus lead to alter the stage group in significant number of cancer patients missed by CECT avoiding unnecessary surgery. Future work should be concentrated on PET/CT to be more routinely used in esophageal cancer for the staging of the disease.

As an important diagnostic method, PET/CT showed a great impact on the pre-treatment evaluation, diagnosing, and staging of patient with esophageal cancer.

\section{Abbreviations \\ 18F-FDG PET/CT: 18F-fluorodeoxyglucose positron emission tomography/ computed tomography; CRC: Colorectal cancer; CTC: Computed tomography colonography; SUV: Standard uptake value}

\section{Acknowledgements \\ Not applicable.}

Committee's reference number

Not applicable.

Author's contributions

The author read and approved the final manuscript.

Funding

None

\section{Availability of data and materials}

The datasets used and/or analyzed during the current study are available from the corresponding author on reasonable request.

\section{Ethics approval and consent to participate}

The protocol of the current study was approved by the ethical scientific committee of October 6 University review board. Written consent was signed prior to perform the examination for each patient.

Consent for publication

Consent for publication was signed for each patient.

\section{Competing interests}

The author declares that there is no competing interests.

Received: 24 September 2019 Accepted: 11 December 2019 Published online: 30 December 2019

\section{References}

1. Njei B, McCarty TR, Birk JW. Trends in esophageal cancer survival in United States adults from 1973 to 2009: A SEER database analysis. J Gastroenterol Hepatol, 2016; 31: 1141-1146. [PMC free article] [PubMed]

2. Walker AJ, Spier BJ, Perlman SB et al (2011) Integrated PET/CT fusion imaging and endoscopic ultrasound in the pre-operative staging and evaluation of esophageal cancer. Mol Imaging Biol 13:166-171 [PubMed]

3. Claire B, Ben H, Glyn G et al (2012) Accuracy of PET/CT in predicting survival in patients with esophageal cancer. World J Surgery 36:1089-1095

4. AJCC (2010) Cancer Staging Manual, 7th edn. Springer Verlag, New York, NY

5. Sjoquist KM, Burmeister BH, Smithers BM et al (2011) Survival after neoadjuvant chemotherapy or chemoradiotherapy for resectable oesophageal carcinoma: an updated meta-analysis. Lancet Oncol 12:681692 [PubMed]

6. Barber TW, Duong CP, Leong T et al (2012) 18F-FDG PET/CT has a high impact on patient management and provides powerful prognostic 
stratification in the primary staging of esophageal cancer: a prospective study with mature survival data. J Nucl Med 53:864-871 [PubMed]

7. Malik V, Johnston C, Donohoe C et al (2012) 18F-FDG PET-detected synchronous primary neoplasms in the staging of esophageal cancer: incidence, cost, and impact on management. Clin Nucl Med 37:1152-1158 [PubMed]

8. Muijs CT, Beukema JC, Pruim J et al (2010) A systematic review on the role of FDG-PET/CT in tumour delineation and radiotherapy planning in patients with esophageal cancer. Radiother Oncol 97:165-171 [PubMed]

9. Kim TJ, Kim HY, Lee KW et al (2009) Multimodality assessment of esophageal cancer: preoperative staging and monitoring of response to therapy 9. Radiographics 29:403-421

10. Kato H, Miyazaki T, Nakajima M et al (2005) The incremental effect of positron emission tomography on diagnostic accuracy in the initial staging of esophageal carcinoma. Cancer 103:148-156 [PubMed]

11. Van Vliet E, Heijenbrok-Kal M, Hunink M et al (2008) Staging investigations for oesophageal cancer: a meta-analysis. Br J Cancer 98:547-557

12. Praveen K,Njshikant A.,Chandrasekhor B.Role of F18-FDG PET/CT in the staging and restaging of esophageal cancer: a comparison with CECT. Indian J.Surg Oncol (2011)2(4):343-350

13. Van Rossum PS, van Lier AL, Lips IM, Meijer GJ et al (2015) Imaging of oesophageal cancer with FDG-PET/CT and MRI. Clin Radiol. 70:81-95

14. Malik V, Harmon M, Johnston C, et al. Whole body MRI in the staging of esophageal cancer-a prospective comparison with whole body 18F-FDG PET-CT. Dig Surg. 2015; 32: 397- 408. CrossrefPubMedWeb of Science.

15. Ying Zhu, Lei Fu, Wang Jing, et al. The value of magnetic resonance imaging in esophageal carcinoma: tool or toy? Asia-Pacific J.Clinical Oncology (2019)15(3):101-107.

\section{Publisher's Note}

Springer Nature remains neutral with regard to jurisdictional claims in published maps and institutional affiliations.

\section{Submit your manuscript to a SpringerOpen ${ }^{\circ}$ journal and benefit from:}

- Convenient online submission

- Rigorous peer review

- Open access: articles freely available online

- High visibility within the field

- Retaining the copyright to your article

Submit your next manuscript at $\boldsymbol{\nabla}$ springeropen.com 